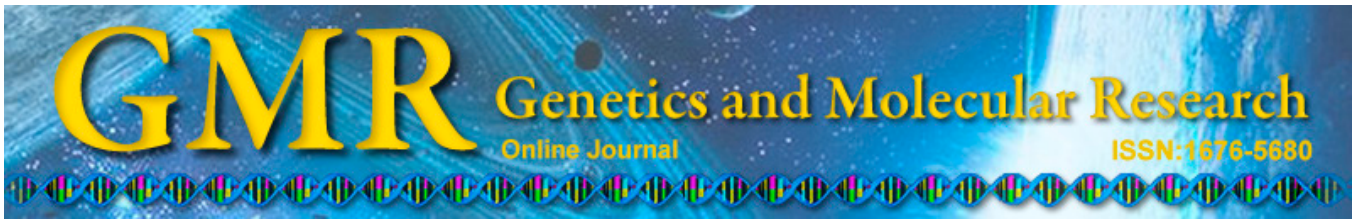

\title{
Structural modeling and analysis of dengue-mediated inhibition of interferon signaling pathway
} S. Khusro ${ }^{3}$, T. Ahmad ${ }^{4}$, S.A. Muhammad ${ }^{5}$, U. Niazi ${ }^{6}$ and V. Azevedo ${ }^{7}$

${ }^{1}$ Atta-ur-Rahman School of Applied Biosciences, National University of Sciences and Technology, Islamabad, Pakistan ${ }^{2}$ Research Center for Modeling \& Simulation, National University of Sciences and Technology, Islamabad, Pakistan ${ }^{3}$ Department of Computer Science, University of Peshawar, Peshawar, Pakistan ${ }^{4}$ Shifa College of Pharmaceutical Sciences, Shifa Tameer-e-Millat University, Islamabad, Pakistan ${ }^{5}$ Institute of Molecular Biology and Biotechnology, Bahauddin Zakariya University Multan, Pakistan ${ }^{6}$ Institute of Biological, Enviromental and Rural Sciences, Aberystwyth University, Aberystwyth, Ceredigion, Wales ${ }^{7}$ Laboratório de Genética Celular e Molecular, Departamento de Biologia Geral, Universidade Federal de Minas Gerais, Belo Horizonte, MG, Brasil

Corresponding authors: J. Ahmad/ V. Azevedo

E-mail: dr.ahmad.jamil@gmail.com / vascoariston@gmail.com

Genet. Mol. Res. 14 (2): 4215-4237 (2015)

Received December 5, 2014

Accepted February 19, 2015

Published April 28, 2015

DOI http://dx.doi.org/10.4238/2015.April.28.4

ABSTRACT. Dengue virus (DENV) belongs to the family Flaviviridae and can cause major health problems worldwide, including dengue fever and dengue shock syndrome. DENV replicon in human cells inhibits interferon $\alpha$ and $\beta$ with the help of its non-structural proteins. Nonstructural protein 5 (NS5) of DENV is responsible for the proteasomemediated degradation of signal transducer and activator of transcription 
(STAT) 2 protein, which has been implicated in the development of resistance against interferon-mediated antiviral effect. This degradation of STAT2 primarily occurs with the help of E3 ubiquitin ligases. Seven in absentia homologue (SIAH) 2 is a host protein that can mediate the ubiquitination of proteins and is known for its interaction with NS5. In this study, comprehensive computational analysis was performed to characterize the protein-protein interactions between NS5, SIAH2, and STAT2 to gain insight into the residues and sites of interaction between these proteins. The objective of the study was to structurally characterize the NS5-STAT2, SIAH2-STAT2, and NS5-SIAH2 interactions along with the determination of the possible reaction pattern for the degradation of STAT2. Docking and physicochemical studies indicated that DENV NS5 may first interact with the host SIAH2, which can then proceed towards binding with STAT2 from the side of SIAH2. These implications are reported for the first time and require validation by wet-lab studies.

Key words: Signal transducer and activator of transcription 2; Dengue virus; Non-structural protein 5; Seven in absentia homologue; Interface residues; Protein-protein interactions

\section{INTRODUCTION}

Dengue virus (DENV) belongs to the family Flaviviridae, genus Flavivirus, and is primarily transferred to humans by the Aedes aegypti mosquito. On the basis of neutralization assays, four serotypes (DENV 1-4) have been discovered (Esteva and Vargas, 2003). In tropical and subtropical areas, DENV infection is a major cause of disease, and according to estimates, 2.5 billion people are at risk of infection, while 50 million people are infected per year (GuhaSapir and Schimmer, 2005). DENV infection is either asymptomatic or may result in a wide range of pathological symptoms, which can be categorized as a mild flu-like syndrome known as dengue fever (DF) (Harris et al., 2000) or dengue hemorrhagic fever characterized by increased vascular permeability and fragility. Against the viral infections, type I interferon (IFN) and innate immune responses are considered the first line of defense. For the establishment of efficient infection, most viruses target these elements to avoid being recognized. Several viruses have been shown to inhibit IFN expression in virus-infected cells, demonstrating an immune evasion strategy (García-Sastre, 2001; Muñoz-Jordán et al., 2003). DENV, like other viruses, is capable of inhibiting IFN production with the help of its non-structural (NS) proteins (Morrison et al., 2012). DENV is capable of antagonizing IFN signaling by targeting various components of related signaling pathways by the help of NS2A, NS4A, NS4B and NS5 (Muñoz-Jordán et al., 2003, 2005; Aslam et al., 2014). In primates, the ability of NS5 protein to bind and degrade signal transducer and activator of transcription (STAT) 2 is one of the most important factors, which contributes to the imperfect host response against DENV (Ashour et al., 2009).

IFN $\alpha$ and $\beta$ signaling starts when these cytokines bind to type 1 interferon receptors (IFNAR1/2), which are located on the surface of infected or nearby cells (Branca and Baglioni, 1981). Subsequently, IFNAR1/2 physically associates with two tyrosine kinases, namely Janus kinase 1 (JAK1) and tyrosine kinase 2 (TyK2), and this interaction results in 
phosphorylation of STAT1 and STAT2 (Darnell et al., 1994; Schindler et al., 2007). The phosphorylated STAT1 and STAT2 subsequently translocate into the nucleus, where they transcribe various IFN responsive elements (ISRE) and induce antiviral effects against the virus (Aaronson and Horvath, 2002) (Figure 1). Various experimental studies in IFNAR knockout mice (KO) STAT2 KO mice have already shown the significance of interferon-dependent immune response for restriction of DENV replication (Ashour et al., 2010).

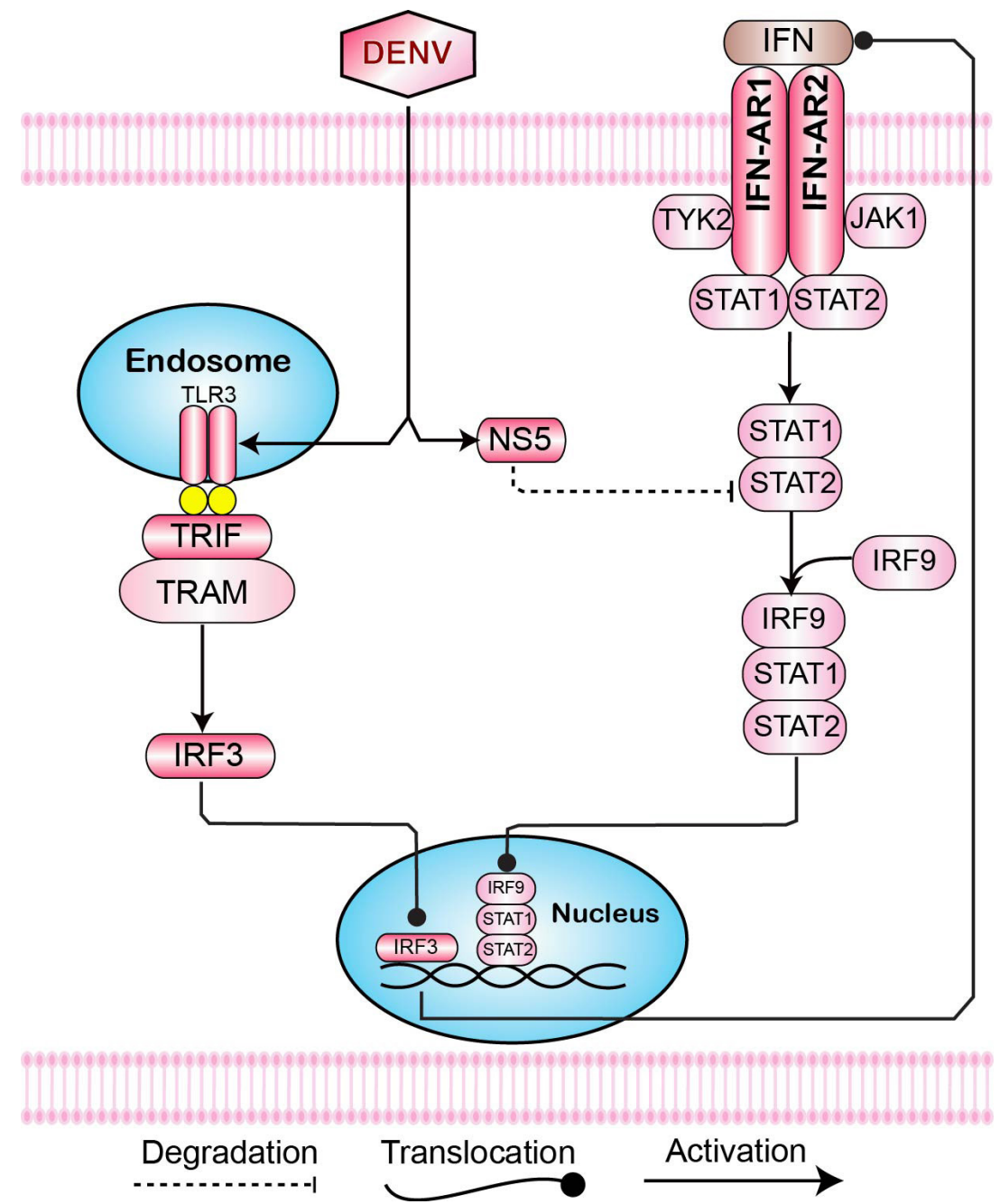

Figure 1. Molecular pathway of DENV mediated inhibition of IFN signaling pathway. Molecular pathway of DENV infection triggers the immune response. DENV enters the cell and triggers the TLR3 pathway (Tsai et al., 2009; Liang et al., 2011). The TLR3 pathway produces IRF3, which in turn transcribe the IFNs (Borden et al., 2007). The IFN then triggers the JAK/STAT pathway through the membrane receptors IFN-AR1 and IFN-AR2 (Abramovich et al., 1994). The function of the JAK/STAT pathway is to induce complex formation between IRF9, STAT1 and STAT2, which will then translocate to the nucleus (Stark et al., 1998; Hu et al., 2008). However, NS5 of DENV interact, in an inhibitory manner, with the STAT2 protein, effectively stopping the IFN triggered JAK/STAT response (Jones et al., 2005; Ashour et al., 2009). 
NS5 protein of DENV antagonizes IFN signaling by binding with STAT2 and causing its subsequent degradation. It has been reported that during the expression of NS5 in the cell, the levels of STAT2 gradually decrease (King et al., 1999; Jones et al., 2005; Ashour et al., 2009). SIAH2 [Uniprot ID: O43255 (human origin)] is an E3 ubiquitin-protein ligase which intercedes ubiquitination $(\mathrm{Ub})$ of target protein and consequent proteasomal degradation (Hu et al., 1997; Habelhah et al., 2002). Le Breton et al. (2011) established the interaction of flavivirus NS3 and NS5 protein with SIAH2, which was later corroborated by the study of Mairiang et al. (2013). These interaction studies encouraged the design of a comprehensive in silico evaluation of NS5, STAT2 and SIAH2 to infer the possible interaction dynamics of the whole system, which is necessary for IFN inhibition and subsequent propagation of DENV pathogenesis.

Accordingly, we performed protein-protein interactions of NS5, STAT2, and SIAH2 in a manner that reflects the possible dynamics involved in the context of reaction mechanisms. STAT2 and SIAH2 were comparatively modeled and optimized to perform protein-protein interactions between them, along with examining the physicochemical properties of the interface areas. Our results indicated that NS5 may first interact with the host SIAH2, which can then proceed towards binding with STAT2 from the side of SIAH2. These implications suggest that DENV hijacks the host proteins to degrade or inhibit specific proteins involved in IFN-mediated signaling pathways, which are necessary to mediate the antiviral effect of IFN.

\section{MATERIAL AND METHODS}

\section{Comparative modeling of STAT2}

The protein sequence of STAT2 (human origin) was obtained from Uniprot (www. uniprot.org) (The UniProt Consortium, 2008) submitted with the ID: P52630. Homologous sequences of the three-dimensional (3D) crystal structures present in the protein database (PDB) (www.rcsb.org/pdb) (Rose et al., 2011) were obtained by submitting the sequence of STAT2 at National Center for Biotechnology Information (NCBI) provided protein specific iteration (PSI)-Blast facility (Altschul and Koonin, 1998). Two structures, PDB-1YVL (crystals structure of phosphorylated STAT-1) (Mao et al., 2005) and PDB-1BF5 (crystal structure of tyrosine phosphorylated STAT1-DNA complex) (Chen et al., 1998) were selected on the basis of the smallest distance on the phylogenetic tree, maximum sequence identities, and the highest positive and the lowest gap values. For the homology modeling of STAT2, these 3-D structures were used as template structures. Expresso (www.tcoffee.org) (Armougom et al., 2006) was used for the sequence alignment of query and template structures of STAT1 and STAT2. Using MODELLER (Eswar et al., 2006), 50 comparative models of STAT2 were generated. On the basis of RMSD values between corresponding residues in each model, comparative structures were clustered by using NMRCLUST (Kelley et al., 1996). For the selection of the representative model, ERRAT (nihserver.mbi.ucla.edu/ERRATv2) (Colovos and Yeates, 1993), Qmean (Benkert et al., 2008), PROSA (Wiederstein and Sippl, 2007), Procheck (Laskowski et al., 1993), and MODELLER provided scores, and potential energy analyses were used as criteria.

\section{Structure of NS5}

The crystal structure of NS5 was obtained from PDB submitted under the ID No. 2J7U (crystal structure of DENV RNA-dependent RNA polymerase catalytic domain resolved 
at $1.85 \AA$ ) (Yap et al., 2007). The sequence of the crystal structure of NS5 (PDB-2J7U) was extracted and PSI-Blast against NCBI Protein Reference Sequences. The sequences having greater than $75 \%$ sequence identity were downloaded and multiple-aligned against the query sequence. Phylogenetic analysis was performed using the default parameters in the MEGA version 6 software (Tamura et al., 2013).

\section{Modeling of SIAH2}

Comparative modeling of SIAH2 was performed since its crystal structure has not yet been resolved. The protein sequence of SIAH2 (human origin) submitted under the Uniprot ID: O43255 was obtained from Uniprot. Homologous sequences of the 3-D crystal structures present in the PDB were obtained by submitting the sequence of SIAH2 at PSI-Blast. Two structures, i.e., PDB-2A25 (crystal structure of SIAH1 SBD bound to the peptide) (Santelli et al., 2005) and PDB-4I7B (SIAH1 bound to synthetic peptide) (Stebbins et al., 2013), were selected using the same criteria as used during the modeling of STAT2. These crystal structures of SIAH1 (PDB IDs: 2A25 and 4I7B) were then used as templates to produce models. Expresso was used for the sequence alignment, and MODELLER was used to generate 50 comparative models of SIAH2. Final selection of the model was performed using the similar criteria and tools as in the comparative modeling of STAT2.

\section{Preparation of structures}

Initial preparation of the protein structures and the complexes obtained after the proteinprotein interactions (PPIs) were prepared for analysis using the Desmond program (Bowers et al., 2006), along with Optimized Potential for Liquid Simulations (OPLS)-AA force field 2005 (Kaminski et al., 2001). The protein preparation wizard was used to prepare the protein structures in terms of generating disulfide bonds, optimization and addition of hydrogen, capping of the C-terminal end and removal of water. The prepared structures were then uploaded in DESMOND system builder. For the MD simulation, the prepared protein molecule was solvated with TIP3P water model in a cubic periodic boundary box, and the system was neutralized by the addition of $\mathrm{Na}^{+}$and $\mathrm{Cl}^{-}$ions. To avoid direct interaction between the proteins' own periodic image, a distance greater than $10 \AA$ between box walls was maintained. For energy minimization of the prepared protein, a required energy minimization step was executed to a maximum of $5 \mathrm{k}$ steps using the steepest descent method until achieving a gradient threshold of $25 \mathrm{kcal} \cdot \mathrm{mol}^{-1} \cdot \AA^{-1}$, followed by L-BFGS (low-memory Broydn-Fletcher-Goldfarb-Shanno quasi-Newtonian minimizer) to meet the $1 \mathrm{kcal} \cdot \mathrm{mol}^{-1} \cdot \AA^{-1}$ convergence threshold. The system was then equilibrated by using the default parameters as given in DESMOND.

\section{Protein-protein interactions}

Guru level interface given at High Ambiguity Driven Bimolecular Docking (HADDOCK) web server (haddock.science.uk.ne) (Dominguez et al., 2003; De Vries et al., 2010) was used to study the protein-protein interactions (PPIs). The approach of the HADDOCK web server is based on the biochemical and/or biophysical interaction data (Liang et al., 2006) along with ab initio PPIs. HADDOCK uses the docking protocol, which supports solvated docking, flexibility of protein regions and modified amino acids. 
Prediction of possible residues of interfaces in each structure was performed by using the CPORT (Concensus Prediction of Interface Residues in Transient) facility (De Vries and Bonvin, 2011). PINuP (Liang et al., 2006), PIER (Kufareva et al., 2007), WHISCY (De Vries et al., 2006), ProMate (Neuvirth et al., 2004), SPPIDER (Porollo and Meller, 2007) and cons-PPISP (Chen and Zhou, 2005) are six interface residues prediction algorithms, which are cumulatively included in CPORT and provide reliable prediction of the interface residues, which can be integrated into the HADDOCK web server as active and passive site residues.

The Visual Molecular Dynamics (VMD) software (Humphrey et al., 1996) was used for the protonation and partial charge assignment of the structures, whereas the Molecular Operating Environment (MOE) software (Chemical computing groups, MOE Software, version 2013) (Inc.) was used for calculation of electrostatic charges. UCSF Chimera (Pettersen et al., 2004) was used to calculate the Kyte-Doolittle hydrophobic scale on the surfaces of the proteins. PyMol (DeLano, 2002) was used to generate the figures of complexes. Swiss PDB viewer (SPDV) (Guex and Peitsch, 1997) was used in sequence-structure analysis.

\section{RESULTS}

\section{Modeling of STAT2}

Comparative modeling of STAT2 protein was performed to evaluate its interaction with NS5 protein of DENV. The crystal structure of STAT1 (PDB ID: 1YVL) was used as template to create the model.

Fifty models of STAT2 were generated by using MODELLER, the calculated energies of which are shown in Figure 2. By using NMRCLUST, these models were clustered into groups. Ten models were selected for further analysis on the basis of the scores provided by ERRAT, QMEAN, and MODELLER. Qmean score for all 10 models were in the range of $\approx 0.5-0.6$, whereas ERRAT scores were in the range of $\approx 70-80$. The best models for preparation were selected on the basis of higher ERRAT scores and lower energy values. Models were re-evaluated for ERRAT and Qmean scores and potential energies after preparation and for final selection.

By superpositioning and multiple sequence alignment (Figure 3) of STAT2 with its template structures, comparable secondary structures of $\mathrm{N}$ domain (residues 1-136) and Core fragment (CF) (residues 137-712) were observed. N domain of STAT1 and 2 was composed of $9 \alpha$-helices, while the CF domain was composed of $15 \alpha$-helices and $21 \beta$-sheets. CF domain was further categorized into 4 sub-domains known as coiled-coil domain (residues 137-317), DNA binding domain (residues 318-488), linker domain (residues 489-576) and SH2 domain (residues 577-683). At the C terminal end, STAT2 had a portion of the phosphorylated tail segment (residue 684-712).

Some of the important regions of STAT1 (PDB ID: 1YVL) and STAT2 are discussed here for inter-comparison in terms of their residual interactions. In the crystallographic study of STAT1, used as template of STAT2, residues 126-133, 181-188, 414-425, 544-550, and 621-625 were not included in the final model due to their disorderness. These residues in our model were energy minimized followed by proper rotamer exploration.

The sequence of residues 126-133 were missing in the crystal structure of STAT1 (PDB ID: 1YVL). These residues of STAT2 model were involved in hydrogen bond interactions between Gln126 with Glu131 and Gln129 with Glu135. A portion of residues ranging from 181-188 were found missing in the crystal structure of STAT1, whereas in the compara- 
tive structure of STAT2, hydrogen bond interactions were observed with Ser187 and Gln192. The region of residues from 414-425 was missing in the crystal structure of STAT1, but in STAT2, these residues were interconnected by hydrogen bonds between Gln371 and Gly416, Gly372 and Ser410, and Ser413 and Ser486. Similarly, within a region of 544-550 residues, salt bridge formation was present between Glu426 and Lys543, which was not found in the structure of STAT1 due to disorderness. Another salt bridge was present with Lys591 and Asp621, whereas Asp621 was also tethered by hydrogen bond interaction with Glu592. Hydrogen bond interaction between Gln618 and Glu653 was also observed within this region.

Mao et al. (2005) reported in a crystallographic study of STAT1 that the front view of STAT1 tetramer is somewhat different from the back view, implying that the connecting regions between $\mathrm{CF}$ and $\mathrm{N}$ domains were not visible due to disorderness. But according to Vinkemeier et al. (1996), the amino acid (aa) sequence (116-142) of this region has a role as flexible tether because of its hydrophobic nature. The model of STAT1 presents the following orientations and interactions of residues which are numbered from 119 to 145 in case of STAT2. In the model of STAT2, hydrogen bond interactions between Gln126 and Glu131, Gln129 and Glu135, Gln142 and Glu146, Gln142 and Gln257, and Glu144 and Arg148, hydrophobic interactions between Met6 and Leu119, Leu7 and Leu119, Leu7 and Leu119, Ile145 and Leu230, and Ile145 and Trp261, and ionic interactions between Glu144 and Arg148 stabilized the structure, and most of the them could be found also in the crystal structure of STAT1.

Mutations Phe77Ala and Leu78Ala have been reported to effect the dimerization of STAT1 N-domain (Chen et al., 2003), whereas in the comparative structure of STAT2, no intermolecular interactions were found for the residues at the same positions. In CF-CF domain dimerization, only three hydrophobic residues were identified, which helps in pointing towards the other molecules by their side chains. On the other hand, STAT2 showed hydrogen bond interactions at the same positions between Leu355 and Thr385, along with the hydrophobic interaction between Leu379 and Val406.

The SH2 subdomain of STAT1 has been implicated as an important site for interacting with the $\alpha$ chain of human interferon (IFN)- $\gamma$, mediated by Arg602, Lys584, His629, and Tyr634 (Mao et al., 2005). Within the structure of STAT2, hydrogen bond interactions between Arg583 and Arg587, Arg601 and Ser613, Arg601 and Ser627, and Glu616 and Tyr626 and a salt bridge between Glu586 and Arg601 restrict the flexibility of this domain.
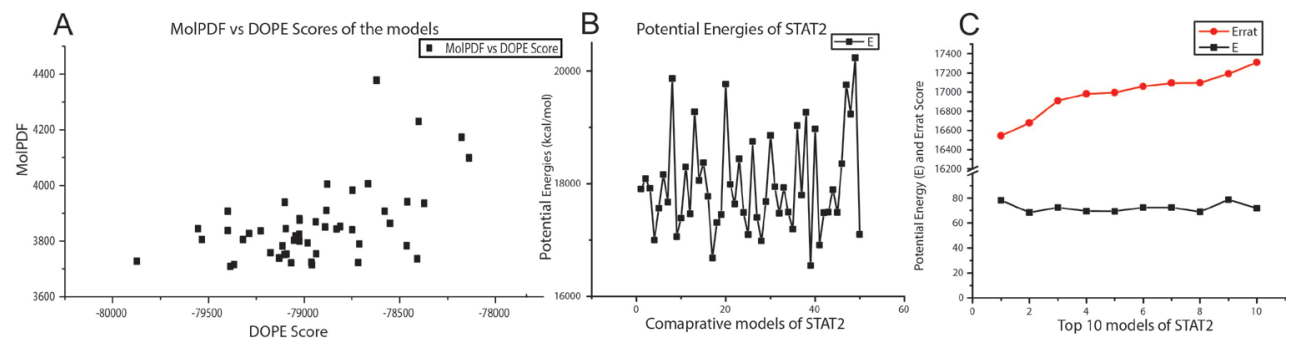

Figure 2. Computed modeling scores and potential energies of comparative models of STAT2. A. This plot shows the comparison of the MODELLER generated MolPDF score and DOPE score of the different homology models. As per the MODELLER and DOPE scores (Eswar et al., 2006), lower MolPDF and lower DOPE scores provide for better quality of the model. B. This plot shows the potential energy values of each of the 50 models. Model No.s 17 and 39 show one of the lowest potential energies, making them better choices for further study compared to the remaining models. C. The subset of 10 favored models, plotted with the potential energies and ERRAT score. 


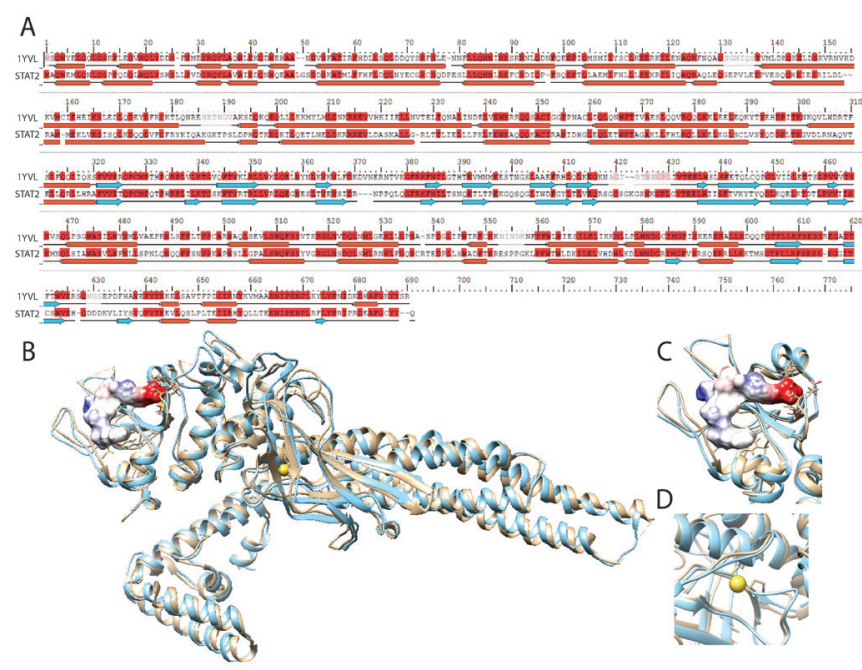

Figure 3. Sequence and structure alignment of STAT2 with template structure of STAT1 (PDB ID: 1VYL). A. Sequence alignment of STAT2 and 1YVL aligning the 2-D protein structures of both sequences. B. Superimposition of the 3-D structure of STAT2, shown in blue, over the template structure of STAT1 with PDB-1YVL, shown in white. The predicted structure of STAT2 almost aligns perfectly with the structure of STAT1. C. Close up view of the inhibitor binding region. D. Close up view of the Zinc binding domain of STAT1.

\section{Modeling of SIAH2}

Comparative modeling of SIAH2 (residues 164-324) was performed to assess its relation with NS5 and STAT2. Crystal structures of SIAH1 (PDB ID: 2A25) was used as template to produce 50 models. In this section, we present insight into the selection of SIAH2 model and its resemblances and contrasts from its template structures.

Figure 4A revealed the MODELLER-reported energies for all models. By using NMRclust these models were clustered into groups, and the models were selected from each cluster. On the basis of ERRAT score, Qmean score, MODELLER-reported energies and potential energies (Figure 4B), 10 models were selected for further analysis (Figure 4C).
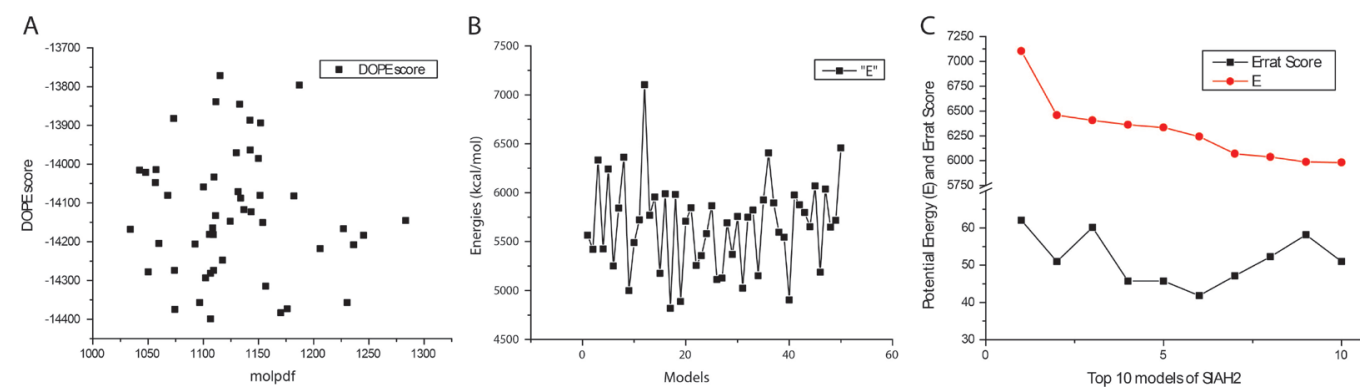

Figure 4. Computed modeling scores and potential energies of comparative models of SIAH2. A. MolPDF vs DOPE score plot of the 50 models of SIAH2 generated by MODELLER. Models with both lower MolPDF and lower DOPE score were selected. B. Potential energy plot of the generated models. Model No.s 18 and 40 shows the least potential energy, making them favored for further analysis over the remaining models. C. Subset of 10 favored models, plotted with the potential energies and ERRA score. 
For all 10 models, the ERRAT scores were in the range of 50-70, while the Qmean score was in the range of 0.1-0.2, so the best model in terms of lower energy values and higher ERRAT and Qmean scores were chosen for preparation. The models were reassessed for the ERRAT score, Qmean score and potential energies after preparation. Final selection of the model was based on the highest ERRAT score, which increased up to $78 \%$, Qmean score, which increased up to $\approx 0.5$, and potential energy, which was relatively lower than those of other models (Figure 4C).

The sequence alignment (Figure 5A) and superposition of comparative structures of SIAH2 with its template showed comparable secondary structures of various domains (Figures 5B and 6). SIAH2 was composed of $4 \alpha$-helices $(\alpha 1-\alpha 4)$ and $9 \beta$-sheets ( $\beta 1-\beta 9)$, whereas SIAH1 comprised $4 \alpha$-helices ( $\alpha 1-\alpha 4)$ and 12 strands of $\beta$-sheets ( $\beta 1-\beta 12)$ (Figure 6).

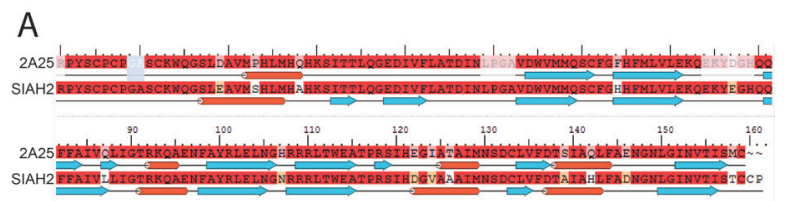

B

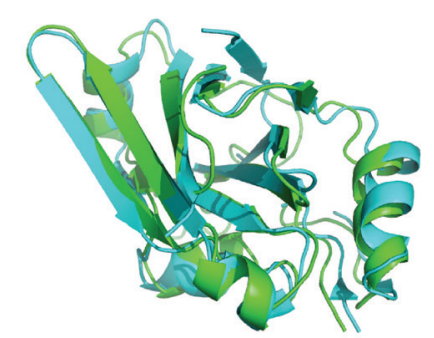

Figure 5. Superimposed structures of SIAH1 (PDB ID: 2A25) and SIAH2. A. Sequence alignment of structure of SIAH1 (PDB-2A25) and comparative model of SIAH2 with 2-D structures. B. 3-D structure alignment of the predicted SIAH2 model (green) with the structure of SIAH1 (PDB-2A25) (blue).

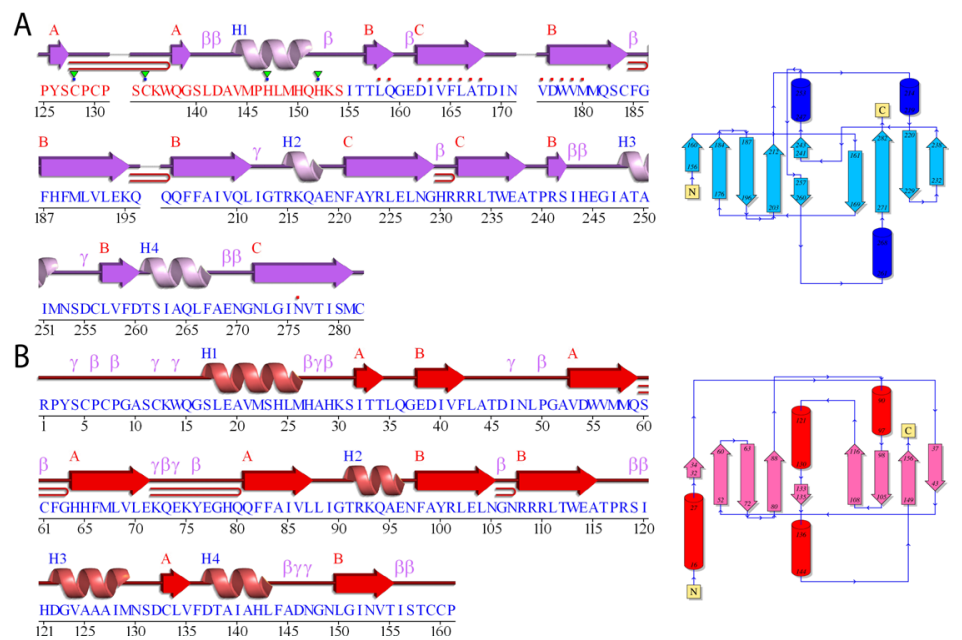

Figure 6. Secondary Structure of SIAH2. Secondary structures and topology diagrams of SIAH1 (A) and SIAH2 (B). 
Most of the intra-molecular interactions of residues in SIAH2 were analogous to the corresponding interactions in its templates. The $\alpha$-helix $\alpha 1$ of SIAH1 shows consensus sequence VMXHLMH (residues 21-27) with other homologous proteins including mmSIAH1A, mmSIAH1B, hsISAH1, hsSIAH2 and xISIAH (Polekhina et al., 2001), also found in modeled protein (SIAH2), i.e., VMXHLMH (residues 21-27). The region KQA (residues 93-95), which lies in the $\alpha$-helix $\alpha 2$ of SIAH1 was also found to be identical in SIAH2, i.e., KQA (residues 93-95). The intra-molecular interactions of both of these domains were found comparable, indicating that the overall construct of the protein model was in agreement with the crystal structure of SIAH1. The $\mathrm{C}$ terminal region is composed of short $\alpha$-helices scattered between the $\beta$-sheets (Polekhina et al., 2001). In comparison, the template structure of SIAH1 and modeled structure of SIAH2 showed the same orientations of $\alpha$-helices and $\beta$-sheets (Figure 6).

In this study, we observed that two salt bridges and 13 hydrogen bonds were involved in forming inter-molecular interactions between dimers of SIAH1, which play a role in retention of the dimer formation (Polekhina et al., 2001). The hydrophobic residues forming the core of the dimer interface include the invariant Phe267 and Trp236 (Polekhina et al., 2001), which were also observed in the modeled structure of SIAH2 at the same positions, forming the comparable intra-molecular interactions with neighbor residues as observed for the respective residues in the crystal structure of SIAH1. The conservatively substituted residues in SIAH2, including Ile263, Leu234, and Val258 (Polekhina et al., 2001), were also observed in the same region present in the crystal structure of SIAH1.

Another important feature of SIAH1 was its Cys-rich region, which formed two zinc fingers. The first zinc finger region was not modeled due to the exclusion of that specific sequence, whereas the second zinc finger was composed of Cys128, Cys135, His174, and His152, making the same intra-molecular contacts with neighbor residues. The strictly conserved regions including Lys153, Arg241, and Cys184 were also observed in the same region of comparative structure of SIAH2, making corresponding hydrogen bond interactions with neighbor residues.

In comparison to the reported protein recognition surfaces (Polekhina et al., 2001), we were able to identify and present the same structural features of SIAH2. In comparison with the template, the modeled structure of SIAH2 also had a shallow groove, which is constructed by the anti-parallel $\beta$-sheets, consisting of strands $\beta 1, \beta 5, \beta 6$, and $\beta 8$.

\section{Protein-protein interactions}

\section{NS5 and STAT2}

The selected model of STAT2 was docked with the crystal structure of NS5 by using default settings of GURU interface provided by the HADDOCK web server.

The predictions for active and passive residues provided by CPORT for STAT2 and NS5 are shown in Figure S1A and $\underline{\mathbf{B}}$, respectively. The HADDOCK web server generated 169 water-refined complexes, which were clustered into 7 groups. The complex with lowest HADDOCK score, $31.3 \pm 9.6$, represented the highest credible complex among other predictions. Other scores of RMSD from the overall lowest energy structure, desolvation energy, van der Waals energy, Z-Score and restraints violation energy for the top 7 clusters are shown in $\underline{\text { Figures S2 }}$ and $\underline{\mathbf{S} 3}$. For improvement of the HADDOCK score, the top cluster was refined for orientation of molecules against each other. 
After refinement of these complexes, the statistics were carried out and are shown in Figure S4. The refined complex of STAT2 and NS5 is shown in Figure 7. After the analysis of deformation of structures in terms of angles, atomic clashes, bond lengths, dihedrals and contact energies of STAT2 and NS5, we found that the structures maintained their quality and consistency after refinement. The analysis of intermolecular interactions between STAT2 and NS5 in the selected complex demonstrated that the refined complex had 28 non-covalent bonding interactions, which included 4 ionic bonds, 17 hydrogen bonds, and 7 hydrophobic interactions between different residues of STAT2 and NS5.

It was observed that helices $\alpha 5$ and $\alpha 8$, loops between helix $\alpha 25$ and strand $\beta 13$, and helices $\alpha 28$ and $\alpha 29$, along with the C terminal of STAT2 were involved in making interactions with NS5. On the other hand, the major domains of NS5 including helices $\alpha 5$ and $\alpha 6$, especially within the regions of Thr346 and Gln350, along with the residues of helix a3, including Asp332, Lys325, Ile320 and Lys329, were found supporting the interaction between NS5 and STAT2.

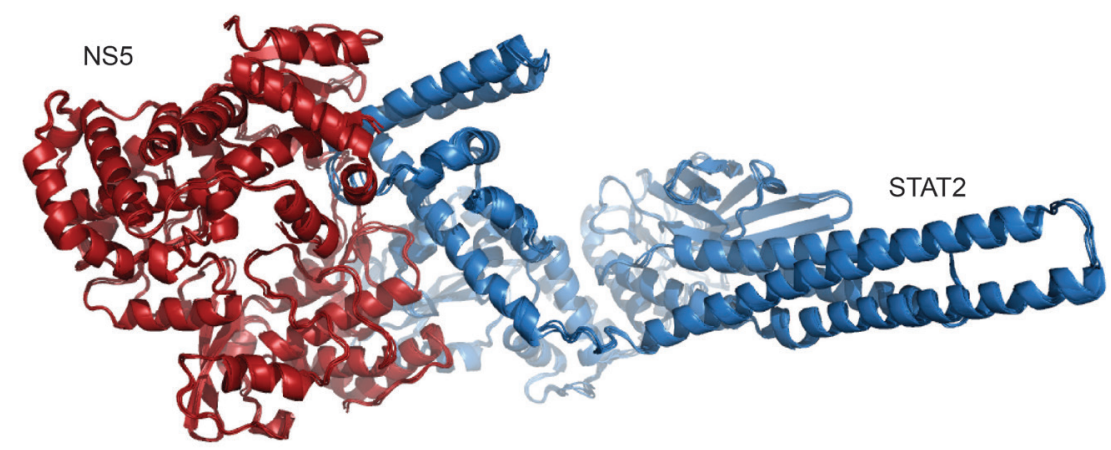

Figure 7. Superimposed refined complex of NS5 and STAT2. Cartoon of STAT2 is shown in blue whereas NS5 is shown in metallic red. The orientation and positioning show how and where the NS5 and STAT2 proteins interact to form complexes.

\section{SIAH2 and STAT2}

The CPORT predictions for active and passive residues of SIAH2 are shown in $\mathbf{\text { Fig- }}$ ure S5. Eighty-one water-refined complexes, clustered into 12 groups representing $40.5 \%$ of the models were given by the HADDOCK web server. The complex with lowest HADDOCK score, $-65.1 \pm 18.96$, represented the highest credible complex among other predictions. Other scores of RMSD from the overall lowest energy structure, desolvation energy, van der Waals energy, Z-score and restraints violation energy for the top 7 clusters are shown in Figures S6 and $\underline{\mathbf{S} 7}$. For improvement of the HADDOCK score, the top cluster was refined for orientation of molecules against each other.

After refinement of the top scoring complex, the statistics were carried out and are shown in Figure S8. Refined complex of STAT2 and SIAH2 has been shown as cartoon in Figure 8. After the analysis of deformation of structures in terms of angles, atomic clashes, bond lengths, dihedrals and contact energies of STAT2 and SIAH2, we found that the structures maintained their quality and consistency after refinement. The analysis of intermolecular interactions between STAT2 and SIAH2 in the selected complex demonstrated that the refined complex had 33 non-covalent bonding interactions, including 14 hydrogen bonds, 8 ionic 
bonds and 9 hydrophobic interactions between different residues of SIAH2 and STAT2, as described below.

Within the N-terminals of SIAH2 and STAT2, hydrophobic interactions were found to be dominant in stabilizing the complex as compared to the hydrogen bonding or ionic interactions. In this aspect, the interactions of Val21, Met22, and Met26 of SIAH2 with Leu49, Phe109 and Leu113 of STAT2 were involved in the hydrophobic interactions, where hydrogen bonding was observed only between Ser17 and Leu25 (residues in $\alpha-1$ helix) of SIAH1 and Gln45 and Arg 117 of STAT2, respectively.

The strands of $\beta$ - sheets ( $\beta-1$ and $\beta-2)$ of SIAH 2 were engaged in making several polar and non-polar contacts with the C-terminal of STAT2, especially within a region of residues from Arg662-Glu672. Among them, the residues of $\beta-3$ sheet of SIAH2 were involved mostly in forming polar contacts with the C-terminal of STAT2. Among them, Asp at positions 52 and 54 of SIAH2 was also involved in making salt bridges with Arg92 and Arg667, which belongs to the two different domains (N-terminal domain and C-terminal domain) of STAT2, respectively. The other interactions (salt bridges) of SIAH2 were mostly found with the C-terminal of STAT2, especially, involving the residues Asp146 and Pro161 of SIAH2 with Hys592, Arg646, and Arg670 of STAT2.

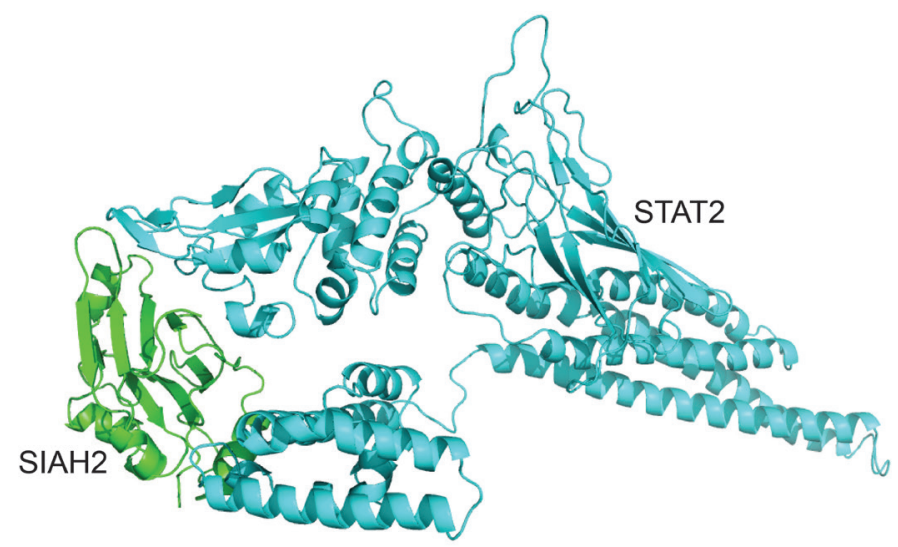

Figure 8. Complex of SIAH2 and STAT2. Cartoon of SIAH2 is shown in green whereas structure of STAT2 is shown in blue. The orientation and positioning show the site of interaction between SIAH2 and STAT2 proteins.

\section{SIAH2 and NS5}

The HADDOCK web server produced 153 water-refined complexes, clustered into 15 groups representing $76.5 \%$ of the models. The complex with the lowest HADDOCK score, $-24.0 \pm 21.7$, represented the highest credible complex among other predictions. Other scores of RMSD from the overall lowest energy structure, desolvation energy, van der Waals energy, Z-score and restraints violation energy for the top 10 clusters are shown in Figures S9 and S10. For improvement of the HADDOCK score, the top cluster was refined for orientation of molecules against each other.

After refinement of the top scoring complex, the statistics were carried out and are shown in Figure S11. The refined complex of NS5 and SIAH2 is shown as a cartoon in Figure 9. After the analysis of deformation of structures in terms of angles, atomic clashes, bond 
lengths, dihedrals and contact energies of NS5 and SIAH2, we found that the structures maintained their quality and consistency after refinement. The analysis of inter-molecular interactions between NS5 and SIAH2 in the selected complex demonstrated that the refined complex had 28 non-covalent bonding interactions, including 16 hydrogen bonds, 3 ionic bonds and 9 hydrophobic interactions between different residues of SIAH2 and NS5, as described below.

Within the $\alpha-1$ domain (at N-terminal) of SIAH2, residues Leu18 and Met22 were involved in a polar interaction with residues Ser747 and Arg749 of NS5, along with hydrophobic interactions with Leu748 and Trp 787 of NS5. These types of interactions tether the Nterminal region of SIAH2 with the C-terminal region of NS5. The region between strands $\beta 1$ and $\beta 2$ of SIAH2 were involved in interactions with the regions composed of Ser420, Ile473, Trp474, and Lys578-Gln580 of NS5, including a salt bridge between Glu38 of SIAH2 and Lys578 of NS5. The structural motif of SIAH2 making the 1st $\beta$-hairpin, between strands $\beta 3$ and $\beta 4$, formed contacts within the regions of Ser317-Met319 and Ala744-Gly745 of NS5. In the third $\beta$-hairpin, between strands $\beta 5$ and $\beta 6, \operatorname{Arg} 109$ formed a salt bridge with Glu309 of NS5, along with a hydrogen bond interaction between Gly106 of SIAH2 and Glu286 of NS5 to further stabilize the complex of the two proteins. The C-terminal of SIAH2 was observed forming contacts within the region of $\beta 9$ with Arg436 and Ile473 of NS5, whereas Asp146 of this region in SIAH2 formed a salt bridge with Arg436.

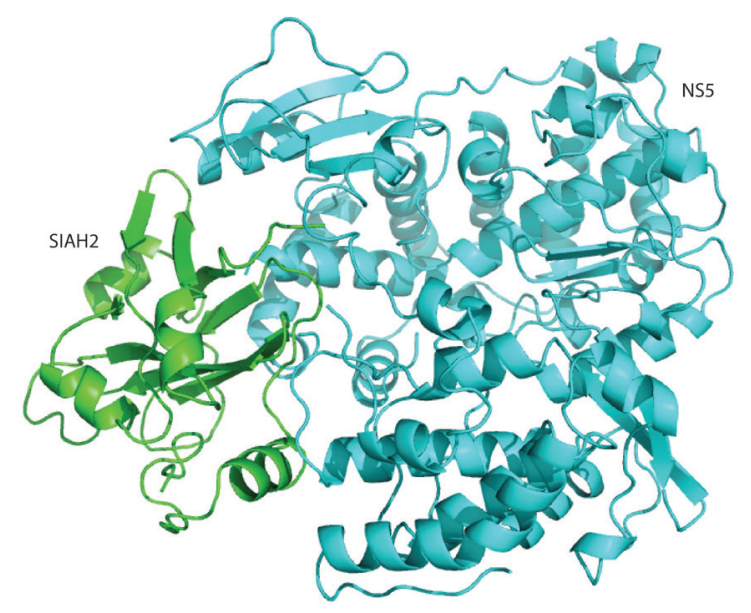

Figure 9. Cartoon of SIAH2 and NS5 complex. Cartoon of NS5 is shown in blue whereas SIAH2 is shown in green. The orientation and positioning show the area of interaction between SIAH2 and NS5 proteins.

\section{Physicochemical characteristics of binding interfaces}

Different methods were utilized to evaluate and support the predictions obtained by the analysis of PPIs, but charge distribution and hydrophobicity at protein surfaces were more effective in validating PPIs. In this section, we explain the physicochemical characteristics of the surfaces of NS5, STAT2, and SIAH2 with focus on the PPI interface area. Electrostatic potential, which was distributed on the surfaces of NS5, STAT2, and SIAH2, was calculated by MOE. Hydrophobicity of these three proteins was analyzed using the Kyte-Doolittle algorithm to further elaborate the dominant nature of interactions, which could be either polar or non-polar. 


\section{Complex of STAT 2 and NS5}

\section{Electrostatic interactions}

Examination of charge complementarity at binding interfaces of NS5 and STAT2 (Figure 10) were found captivating, which help in stabilizing the PPI of STAT2 with NS5 at specific areas as discussed previously. Binding interfaces of STAT2 had strong charge complementarity (Figure $10 \mathrm{~A}$ ), in which $\mathrm{Gln} 45$ was found with strong negative charge around the region, increasing its interaction with NS5 in the region of Ser420 with strong positive charge at its binding interface. Residues of STAT2 including Asp53 and Thr102 formed a heterogeneous negatively charged region, which electrostatically stabilized the complex after forming interactions with the positively charged region of Thr346 in NS5. His62 of STAT2, located near the N terminus, also had a negatively charged region, which promoted binding with Glu286 of NS5 due to its positive charge distribution.

Significant electrostatic charge complementarity was found between the positively charged residues of STAT2, including Glu106, Leu113, Arg117, Asn110, and Phe109, and negatively charged region of NS5, around the areas of Ala744, Trp746, Gly745, Ala744, and Ser420. However, the residue Gln45 of STAT2 was found to have a positively charged region, showing its affinity for the very positively charged surface of Ser420 in NS5, which helps to further stabilize the conformations of the complexed proteins.
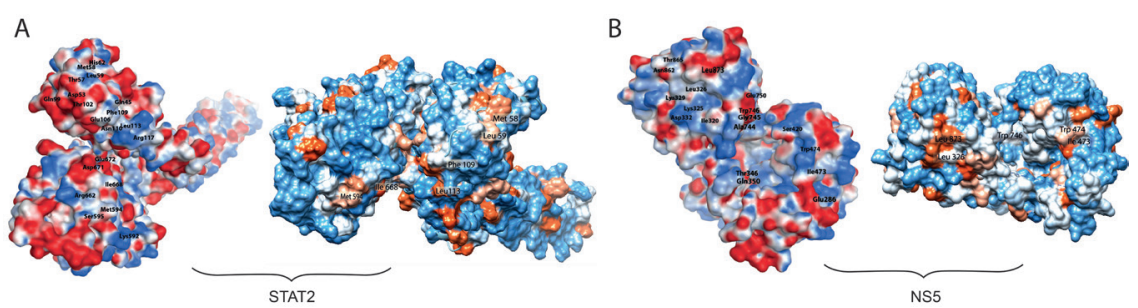

Figure 10. Electrostatic potential at the surfaces of NS5 and STAT2. (A) Electrostatic charge distribution at the surface of STAT2 whereas (B) represents the electrostatic charge distribution at the surface of NS5. Charge compatible residues are labeled on the interaction surface of both proteins.

\section{Hydrophobic interactions}

Hydrophobicity of protein interfaces is presented in Figure 10 for the complex of NS5 and STAT2. In the STAT2 and NS5 complex, STAT2 mostly showed polar regions for interaction with non-polar regions of NS5. The residue Met594 of STAT2 showed hydrophobic interaction with Leu326 located near the non-polar region of NS5. The residue Ile668 of STAT2 promoted a stronger interaction by being involved with Leu326 and Leu873 of NS5, where both of these residues are located in close proximity to stabilize the interactions of the complex. Moreover, Met58 of STAT2 is located in the polar region and it showed interaction with Ile473 of NS5, positioned in the non-polar region of the complex.

\section{Complex of SIAH2 and STAT2}

\section{Electrostatic interactions}

The charge complementarity at binding interfaces of SIAH2 and STAT2 (Figure 11) 
validated the PPI interface areas between these two proteins. The binding interfaces of SIAH2 and STAT2 could be divided into three complementarity charged areas with each other. Divisions on the basis of charges can be seen in Figure 11A and B from top to bottom, for example, where the top portion of SIAH2 is positively charged, middle portion is negatively charged and the bottom portion of the protein is again positively charged. At the top of SIAH2, residues Ser17, Gln59, Arg92 showed a positive charge. On the other hand, this area interacted with the negatively charged area of Asp519 and Arg662 of STAT2. In the middle, SIAH2 had negative charges around the region of Glu38 and Pro161, and this area interacted with the positively charged region of Arg667 and Phe663 of STAT2. At the bottom of SIAH2, the positively charged region around Arg 108 exhibited strong charge complementarity with Asp52 of STAT2.
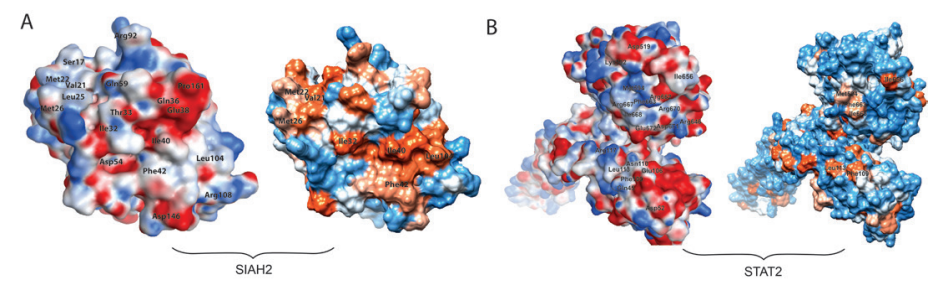

Figure 11. Electrostatic potential and hydrophobicity at the surfaces of SIAH2 and STAT2. (A) Represents the electrostatic charge distribution (left) and hydrophobicity (right) at the surface of SIAH2 whereas (B) represents the electrostatic charge distribution (left) and hydrophobicity (right) at the surface of STAT2. Charge compatible residues are labeled on the interaction surface of both proteins.

\section{Hydrophobic interactions}

The hydrophobic surface of STAT2 is comparatively unexposed, whereas in SIAH2, various hydrophobic residues were found on the PPI interface (Figure 11). Residues Val21, Met22, Met26, Ile32, Ile40, Phe42, and Leu104 of SIAH2 formed hydrophobic interactions especially with the regions of Phe109, Ile656, Phe663, and Ile668 in STAT2.

\section{Complex of SIAH2 and NS5}

\section{Electrostatic interactions}

Similar to the above mentioned charge distribution analysis, charge complementarity at the binding interfaces of SIAH2 and NS5 validated the PPI interface areas between the two proteins (Figure 12). As shown in the figure, the structural region in the nearby area of Cys 160 in SIAH2 was found to be attracted towards Trp474 of NS5. In addition, the complex seemed to be stabilized by the intra-molecular interactions with the regions of His64 in SIAH2 and Ser747 in NS5, which were densely populated with opposite charges.

\section{Hydrophobic interactions}

The hydrophobic surface in NS5 is comparatively unexposed, whereas in SIAH2, various patches of hydrophobic surface can be found (Figure 12). Residues Leu18, Met22, Leu25, Ile40, and Phe62 of SIAH2 played an important role in forming hydrophobic interactions especially with the regions of Leu748 and Ile473 in NS5. 


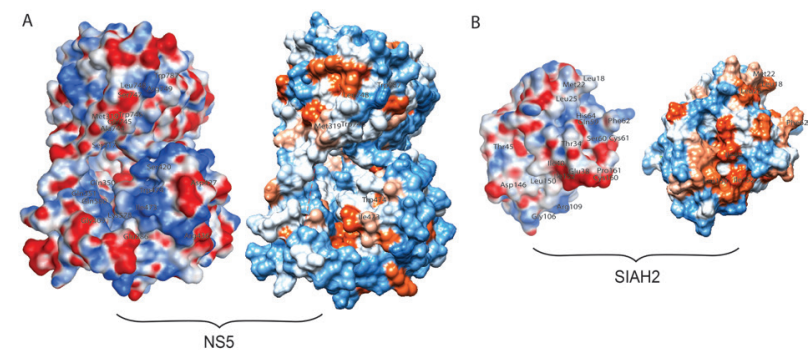

Figure 12. Electrostatic potential and hydrophobicity at the surfaces of SIAH2 and NS5. (A) Represents the electrostatic charge distribution (left) and hydrophobicity (right) at the surface of NS5 whereas (B) represents the electrostatic charge distribution (left) and hydrophobicity (right) at the surface of SIAH2. Charge compatible residues are labeled on the interaction surface of both proteins.

\section{Sequence and phylogenetic analysis of NS5}

The comparison of interface residues of NS5, forming interactions with SIAH2 and/ or STAT2, were analyzed by multiple sequence alignment of NS5 sequences obtained from different species (Figure 13). The analysis of sequence alignment along with the evolutionary relationships (Figure 14) between them promote the conservation of NS5 interface residues predicted in this study. Along with the fact that predicted interface residues are mostly conserved in different serotypes of DENV, it was also observed that the change in a residue often maintained the physicochemical property of the amino acids except in some cases.

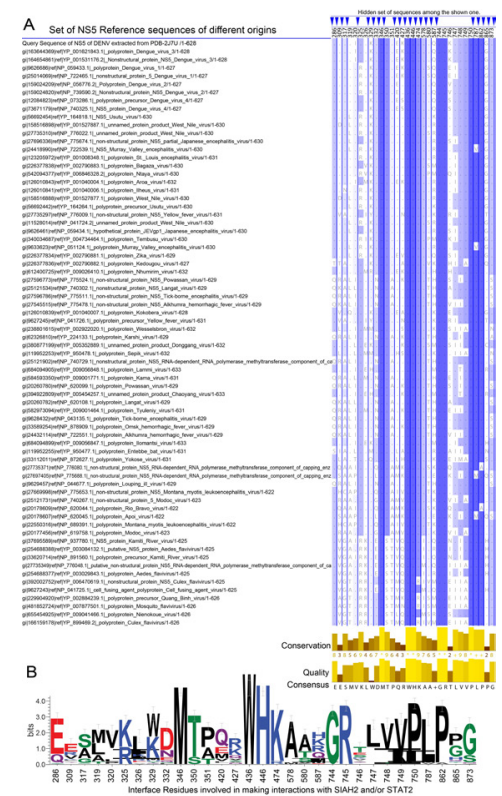

Figure 13. Multiple alignment and consensus sequence logo of NS5 obtained from different origins. (A) Multiple sequence alignment of interface residues of NS5 with other reference sequences obtained from different origins. Intensity of blue color represents the conservation of residues at specific positions; (B) consensus sequence logo was generated for the residues of NS5 involved in making interactions with SIAH2 and STAT2 by using WebLogo (Schneider and Stephens, 1990; Crooks et al., 2004). 


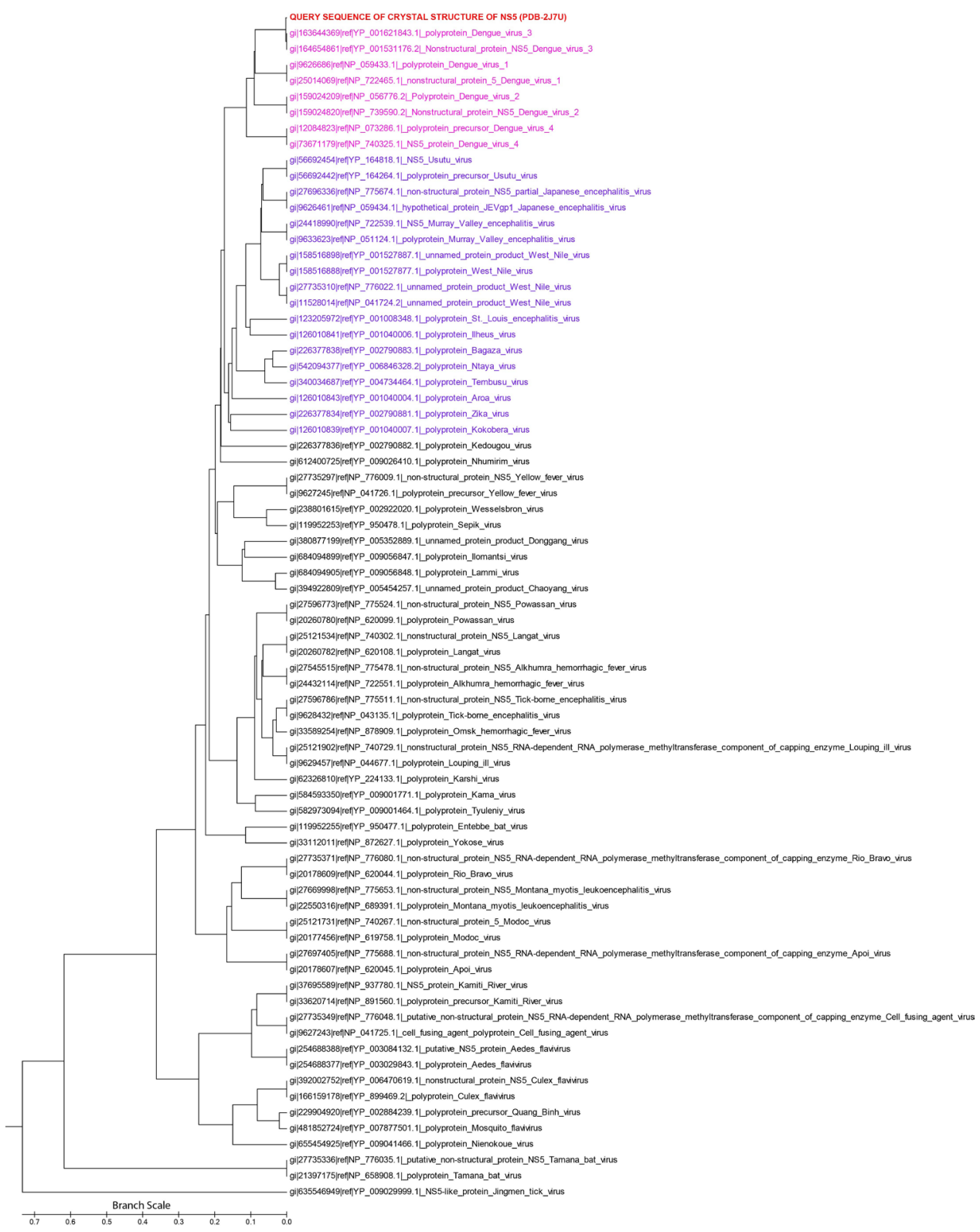

Figure 14. Evolutionary relationships of NS5 protein in different origin. The evolutionary history was inferred using the UPGMA method (Sneath and Sokal, 1973). The optimal tree with the sum of branch length $=7.82765610$ is shown. The tree is drawn to scale, with branch lengths in the same units as those of the evolutionary distances used to infer the phylogenetic tree. The evolutionary distances were computed using the Poisson correction method (Zuckerkandl and Pauling, 1965) and are in the units of the number of amino acid substitutions per site. The analysis involved 75 amino acid sequences. All positions containing gaps and missing data were eliminated. There were a total of 549 positions in the final dataset. Evolutionary analyses were conducted in MEGA6 (Tamura et al., 2013). Query sequence is colored red whereas the most related sequences of NS5 of different origins are colored pink and purple depending upon the distance from query sequence. Others are shown in black having variations in the interface residues. 


\section{DISCUSSION}

The antiviral IFN response plays a role in restricting the replication and dissemination of DENV as one of the first lines of host immune defense (Diamond et al., 2000). To ensure its survival and replication, DENV antagonizes both the synthesis and signaling of IFN and ultimately results in the downregulation of the IFN signaling pathways (Muñoz-Jordán et al., 2003; Jones et al., 2005; Ashour et al., 2009; Rodriguez-Madoz et al., 2010). Previous studies have shown that flaviviruses have evolved UB-dependent strategies to inhibit/degrade STAT proteins (Muñoz-Jordán et al., 2005; Jones et al., 2005; Ashour et al., 2009). NS5 protein of DENV antagonizes the downstream signaling of the IFN- $\alpha / \beta$-dependent JAK/STAT pathway by binding with and subsequently degrading STAT2 (Darnell et al., 1994; Jones et al., 2005). Interestingly, Ashour et al. (2009) demonstrated that the degradation of, but not binding to, STAT2 is performed by NS5 after its proteolytic cleavage from DENV polyprotein and vice versa. Moreover, in the same study, it was discussed that ubiquitin protein ligase E3 component n-recognin 4 (UBR4) is required for the degradation of STAT2. However, UBR4 does not contain a known E3 ligase motif, i.e., homology to E6AP-binding protein C-terminus (HECT) or RING domain (Tasaki et al., 2005), and its role as an E3 ligase for the degradation of STAT2 is still in question (Morrison and García-Sastre, 2014). Among other host proteins that can interact with NS5 of DENV, a member of human seven in absentia homolog, i.e., SIAH2, has also been reported forming interactions with NS5 (Le Breton et al., 2011; Khadka et al., 2011; Mairiang et al., 2013). SIAH2 protein is an E3 ligase and is involved in ubiquitination and proteosome-mediated degradation of specific proteins.

In this study, for the first time, SIAH2 was shown to form a biologically plausible complex with STAT2 depending on the HADDOCK and potential energy scores of the complex, which require wet-lab experiments to confirm the prediction. This prediction is further supported by the fact that SIAH1 has been found to form interactions with STAT3 of human origin (Wang et al., 2011). Moreover, the objective of this study was to see if NS5 protein of DENV promoted the interaction of SIAH2 with STAT2, which possibly leads towards the degradation of STAT2. This study implied that the identification of possible PPIs between NS5, SIAH2 and STAT2 are important to show DENV-mediated STAT2 degradation and may lay the foundation for designing therapeutics that target the NS5/SIAH2 interaction.

In the present study, we used molecular modeling along with in silico PPIs and charge distribution procedures to characterize the structural features of DENV and human host proteins, which can ultimately result in NS5-mediated STAT2 degradation and inhibition of IFN signaling. Comparative modeling of the STAT2 and SIAH2 were performed to deduce the interactions with already present crystal structure of NS5. PPIs between two proteins were used to produce bimolecular first-order reactions, which will be necessary as a first instance of STAT2 degradation. The electrostatic charge distribution and hydrophobic surface analysis of the proteins were used to validate the PPI interfaces along with correlating the scores provided for PPIs and physically plausible sites of interaction. The procedure for the preparation of proteins was adapted using our previous study (Paracha et al., 2014), which produced plausible results. Protein preparation produced relative energy-minimized structures, which were speculated as the final product after a transition state obtained through the modeling procedures or through PPIs.

The quality of comparative protein modeling is connected to the percent sequence identity of query and template sequences (Daga et al., 2010). The accurate homology models 
have nearly $1 \AA$ root mean square error (RMSE) for the atoms in the backbone. They can be correlated with a structure determined with a medium resolution nuclear magnetic resonance (NMR), which needs nearly $50 \%$ identity of the sequence of the query to its template (Baker and Sali, 2001). Homology modeling of biological entities is a more efficient, accurate, and reliable method than other experimental methods (Kryshtafovych et al., 2014). The sequence of STAT2 had $44 \%$ sequence identity with $63 \%$ positives and 2 gaps, when compared with the sequence of the crystal structure of its template structure of STAT1, indicating that the homology model of STAT2 utilized in this study produced noteworthy and insightful results. Several parameters such as Ramachandran, ERRAT, Qmean, PROSA and PROCHECK, were utilized to support the quality and reliability of the final choice of homology model of STAT2. Geometry of the models was also verified during each phase of pre-experimental activity for improvement of backbone, orientation of side chains and rotamers of residues and to decrease the clashes within atoms. The final selected homology model of STAT2 had bond angles, dihedrals, lengths and interaction energies within permitted limits in the absence of any atomic collisions. Furthermore, 0.579 Qmean score, 74\% ERRAT score and associated reduced potential energy were some of the other features that convinced us to utilize the final chosen homology model of STAT2 in PPIs.

Similarly, the sequence of SIAH2 had $87 \%$ sequence identity with $94 \%$ positives and no gaps as compared to the sequence of the crystal structure of its template structure of SIAH1, indicating the reliability of the homology model of SIAH2 in this study. The same set of structural analysis, as used in STAT2 modeling, was also employed in the case of homology modeling of SIAH2, which not only helped to further tune the model but also validated its use in this study. ERRAT score $\approx 78 \%$ and other scoring algorithms assured to the use of the final selection of homology model of SIAH2 in further analysis.

PPIs at the molecular level are important to regulate the different activities of cell and the quality of life. Main information such as the flexibility of structures, binding area and residual interactions are important to understand the mechanisms behind the diseases and to design new therapeutic strategies. Sometimes, complications of experimental methods and techniques restrict the ability to determine the structures of protein-protein complexes. The methods of computational modeling can effectively be used to overcome the restrictions present in the wet-lab. Our present study was an effort to predict the possible binding interfaces involved in the PPI of SIAH2, NS5 and STAT2 with each other and to investigate the role of SIAH2 as ubiquitin protein employed by NS5 for the degradation of STAT2. The availability of the already resolved crystal structure of NS5 in addition to the development of reliable models of STAT2 and SIAH2 allowed us to study the interaction interfaces, non-covalent interactions, shape complementarity, and physicochemical analysis of the proteins.

The interactions of NS5 and STAT2 showed specificity in shape complementarity of the molecules against each other. However, on the basis of the HADDOCK-provided scores and potential energies of the complexes, the binding of NS5 with STAT2 was observed to be weaker in terms of associations between them as compared to the interaction between NS5 and SIAH2. Moreover, the interfaces of SIAH2 and STAT2 in the final selected complex showed numerous hydrophobic, hydrogen bond and ionic bond interactions along with electrostatic charge complementarity observed with better PPI scores compared to any other complex studied here. This implies that NS5 may form a complex with SIAH2 at first instance with subsequent interaction of SIAH2 with STAT2. PPI analysis of SIAH2 and STAT2 demonstrated that this interaction may play a competitive role in the ubiquitination of STAT2. The selected 
complex had closer proximity and greater buried surface area as compared to other complexes studied here. Electrostatic studies demonstrated that the non-covalent interaction resulted in increased attraction between the compatible charged residues of SIAH2 and STAT2.

\section{CONCLUSION}

This structural analysis revealed the association between NS5, SIAH2, and STAT2, which are explicitly involved in interaction with each other. Additional wet-lab mutational analysis of the proteins may provide the grounds for the categoric affiliation of STAT2 with interacting E3 ligases such as SIAH2 and NS5. Nevertheless, the results demonstrated here present essential information about residual interaction between NS5, SIAH2, and STAT2 for understanding the interactions; however, this study was a crucial step towards revealing the interface residues and binding pattern of proteins, which can be used in designing new therapeutic strategies against NS5- and SIAH2-mediated degradation of STAT2. In the future, we would like to conduct wet-lab studies of point mutations at the interfaces of NS5, SIAH2, and STAT2, implicated in this study, to further enhance our understanding of the interaction pattern of NS5-mediated inhibition of IFN signaling through the degradation of STAT2, with the long term goal of finding and targeting similar interaction patterns in other viral species with the conserved NS5 protein.

\section{Supplementary material}

\section{REFERENCES}

Aaronson DS and Horvath CM (2002). A road map for those who don't know JAK-STAT. Science 296: 1653-1655.

Abramovich C, Shulman L, Ratovitski E, Harroch S, et al. (1994). Differential tyrosine phosphorylation of the IFNAR chain of the type I interferon receptor and of an associated surface protein in response to IFN-alpha and IFN-beta. EMBO J. 13: 5871.

Altschul SF and Koonin EV (1998). Iterated profile searches with PSI-BLAST - a tool for discovery in protein databases. Trends Biochem. Sci. 23: 444-447.

Armougom F, Moretti S, Poirot O, Audic S, et al. (2006). Expresso: automatic incorporation of structural information in multiple sequence alignments using 3D-Coffee. Nucleic Acids Res. 34 (Suppl 2): W604-W608.

Ashour J, Laurent-Rolle M, Shi P-Y and García-Sastre A (2009). NS5 of dengue virus mediates STAT2 binding and degradation. J. Virol. 83: 5408-5418.

Ashour J, Morrison J, Laurent-Rolle M, Belicha-Villanueva A, et al. (2010). Mouse STAT2 restricts early dengue virus replication. Cell Host Microbe 8: 410-421.

Aslam B, Ahmad J, Ali A, Paracha RZ, et al. (2014) On the modelling and analysis of the regulatory network of dengue virus pathogenesis and clearance. Comput. Biol. Chem. 53: 277-291.

Baker D and Sali A (2001). Protein structure prediction and structural genomics. Science 294: 93-96.

Benkert P, Tosatto SC and Schomburg D (2008). QMEAN: A comprehensive scoring function for model quality assessment. Proteins Struct. Funct. Bioinformatics 71: 261-277.

Borden EC, Sen GC, Uze G, Silverman RH, et al. (2007). Interferons at age 50: past, current and future impact on biomedicine. Nat. Rev. Drug Discov. 6: 975-990.

Bowers K J, Chow E, Xu H, Dror R O, et al. (2006). Scalable algorithms for molecular dynamics simulations on commodity clusters. In: SC 2006 Conference, Proceedings of the ACM/IEEE. IEEE, 43-43.

Branca AA and Baglioni C (1981). Evidence that types I and II interferons have different receptors. Nature, 294: $768-770$.

Chen $\mathrm{H}$ and Zhou H-X (2005). Prediction of interface residues in protein-protein complexes by a consensus neural network method: test against NMR data. Proteins Struct. Funct. Bioinformatics 61: 21-35.

Chen X, Vinkemeier U, Zhao Y, Jeruzalmi D, et al. (1998). Crystal structure of a tyrosine phosphorylated STAT-1 dimer bound to DNA. Cell 93: 827-839.

Chen X, Bhandari R, Vinkemeier U, van den Akker F, et al. (2003). A reinterpretation of the dimerization interface of the 
N-terminal domains of STATs. Protein Sci. 12: 361-365.

Colovos C and Yeates TO (1993). Verification of protein structures: patterns of nonbonded atomic interactions. Protein Sci. 2: 1511-1519.

Crooks GE, Hon G, Chandonia J-M and Brenner SE (2004). WebLogo: a sequence logo generator. Genome Res. 14: 1188-1190.

Daga PR, Patel RY and Doerksen RJ (2010). Template-based protein modeling: recent methodological advances. Curr. Top Med. Chem. 10: 84-94.

Darnell JE, Kerr IM and Stark GR (1994). Jak-STAT pathways and transcriptional activation in response to IFNs and other extracellular signaling proteins. Science 264: 1415-1421.

DeLano WL (2002). PyMOL. DeLano Scientific, San Carlos, CA, 700.

De Vries SJ and Bonvin AM (2011). CPORT: a consensus interface predictor and its performance in prediction-driven docking with HADDOCK. PLoS One 6: e17695.

De Vries SJ, van Dijk AD and Bonvin AM (2006). WHISCY: What information does surface conservation yield? Application to data-driven docking. Proteins Struct. Funct. Bioinformatics 63: 479-489.

De Vries SJ, van Dijk M and Bonvin AM (2010). The HADDOCK web server for data-driven biomolecular docking. Nat. Protocols 5: 883-897.

Diamond MS, Roberts TG, Edgil D, Lu B, et al. (2000). Modulation of dengue virus infection in human cells by alpha, beta, and gamma interferons. J. Virol. 74: 4957-4966.

Dominguez C, Boelens R and Bonvin A M (2003). HADDOCK: a protein-protein docking approach based on biochemical or biophysical information. J. Am. Chem. Soc. 125: 1731-1737.

Esteva L and Vargas C (2003). Coexistence of different serotypes of dengue virus. J. Math. Biol. 46: 31-47.

Eswar N, Webb B, Marti-Renom MA, Madhusudhan M, et al. (2006). Comparative protein structure modeling using Modeller. Curr. Protocol. Bioinformatics 5.6: 1-30.

García-Sastre A (2001). Inhibition of interferon-mediated antiviral responses by influenza A viruses and other negativestrand RNA viruses. Virology 279: 375-384.

Guex N and Peitsch M C (1997). SWISS-MODEL and the Swiss-Pdb Viewer: an environment for comparative protein modeling. Electrophoresis 18: 2714-2723.

Guha-Sapir D and Schimmer B (2005). Dengue fever: new paradigms for a changing epidemiology. Emerg Themes Epidemiol 2: 1-10.

Habelhah H, Frew I J, Laine A, Janes P W, et al. (2002). Stress-induced decrease in TRAF2 stability is mediated by Siah2. The EMBO J. 21: 5756-5765

Harris E, Videa E, Pérez L, Sandoval E, et al. (2000). Clinical, epidemiologic, and virologic features of dengue in the 1998 epidemic in Nicaragua. Am. J. Trop. Med. Hyg. 63: 5-11.

Hu G, Zhang S, Vidal M, La Baer J, et al. (1997). Mammalian homologs of seven in absentia regulate DCC via the ubiquitin-proteasome pathway. Genes Development 11: 2701-2714.

$\mathrm{Hu}$ X, Chakravarty SD and Ivashkiv LB (2008). Regulation of interferon and Toll-like receptor signaling during macrophage activation by opposing feedforward and feedback inhibition mechanisms. Immunol. Rev. 226: 41-56.

Humphrey W, Dalke A and Schulten K (1996). VMD: visual molecular dynamics. J. Mol. Graph. 14: 33-38.

Jones M, Davidson A, Hibbert L, Gruenwald P, et al. (2005). Dengue virus inhibits alpha interferon signaling by reducing STAT2 expression. J. Virol. 79: 5414-5420

Kaminski GA, Friesner RA, Tirado-Rives J and Jorgensen WL (2001). Evaluation and reparametrization of the OPLS-AA force field for proteins via comparison with accurate quantum chemical calculations on peptides. J. Phys. Chem. B 105: 6474-6487.

Kelley LA, Gardner SP and Sutcliffe MJ (1996). An automated approach for clustering an ensemble of NMR-derived protein structures into conformationally related subfamilies. Protein Engineering 9: 1063.

Khadka S, Vangeloff AD, Zhang C, Siddavatam P, et al. (2011). A physical interaction network of dengue virus and human proteins. Mol. Cell. Proteomics 10: M111.012187

King AD, Nisalak A, Kalayanrooj S, Myint K, et al. (1999). B cells are the principal circulating mononuclear cells infected by dengue virus 30: 718-28.

Kryshtafovych A, Moult J, Bales P, Bazan J F, et al. (2014). Challenging the state of the art in protein structure prediction: Highlights of experimental target structures for the 10th Critical Assessment of Techniques for Protein Structure Prediction Experiment CASP10. Proteins Struct. Funct. Bioinformatics 82: 26-42

Kufareva I, Budagyan L, Raush E, Totrov M, et al. (2007). PIER: protein interface recognition for structural proteomics. Proteins Struct. Funct. Bioinformatics 67: 400-417

Laskowski RA, MacArthur MW, Moss DS and Thornton JM (1993). PROCHECK: a program to check the stereochemical quality of protein structures. J. Appl. Crystallogr. 26: 283-291. 
Le Breton M, Meyniel-Schicklin L, Deloire A, Coutard B, et al. (2011). Flavivirus NS3 and NS5 proteins interaction network: a high-throughput yeast two-hybrid screen. BMC Microbiol. 11: 234.

Liang S, Zhang C, Liu S and Zhou Y (2006). Protein binding site prediction using an empirical scoring function. Nucleic Acids Res. 34: 3698-3707.

Liang Z, Wu S, Li Y, He L, et al. (2011). Activation of Toll-like receptor 3 impairs the dengue virus serotype 2 replication through induction of IFN- $\beta$ in cultured hepatoma cells. PloS One 6: e23346.

Mairiang D, Zhang H, Sodja A, Murali T, et al. (2013). Identification of new protein interactions between dengue fever virus and its hosts, human and mosquito. PloS One 8: e53535.

Mao X, Ren Z, Parker GN, Sondermann H, et al. (2005). Structural bases of unphosphorylated STAT1 association and receptor binding. Mol. Cell 17: 761-771.

Molecular Operating Environment (MOE) (2013). Chemical Computing Group Inc., 1010 Sherbook St. West, Swite \#910, Montreal, H3A 2R7; 2015.

Morrison J and García-Sastre A (2014). STAT2 signaling and dengue virus infection. JAK-STAT 3: e27715.

Morrison J, Aguirre S and Fernandez-Sesma A (2012). Innate immunity evasion by Dengue virus. Viruses 4: 397-413.

Muñoz-Jordán JL, Sánchez-Burgos GG, Laurent-Rolle M and García-Sastre A (2003). Inhibition of interferon signaling by dengue virus. Proc. Natl. Acad. Sci. 100: 14333-14338.

Muñoz-Jordán JL, Laurent-Rolle M, Ashour J, Martínez-Sobrido L, et al. (2005). Inhibition of alpha/beta interferon signaling by the NS4B protein of flaviviruses. J. Virol. 79: 8004-8013.

Neuvirth H, Raz R and Schreiber G (2004). ProMate: a structure based prediction program to identify the location of protein-protein binding sites. J. Mol. Biol. 338: 181-199.

Paracha RZ, Ali A, Ahmad J, Hussain R, et al. (2014). Structural evaluation of BTK and PKC-d mediated phosphorylation of MAL at positions Tyr86 and Tyr106. Comput. Biol. Chem. 51: 22-35.

Pettersen EF, Goddard TD, Huang CC, Couch GS, et al. (2004). UCSF Chimera - a visualization system for exploratory research and analysis. J. Comput. Chem. 25: 1605-1612.

Polekhina G, House CM, Traficante N, Mackay JP, et al. (2001). Siah ubiquitin ligase is structurally related to TRAF and modulates TNF- $\alpha$ signaling. Nat. Struct. Mol. Biol. 9: 68-75.

Porollo A and Meller J (2007). Prediction-based fingerprints of protein-protein interactions. Proteins Struct. Funct. Bioinformatics 66: 630-645.

Rodriguez-Madoz JR, Bernal-Rubio D, Kaminski D, Boyd K, et al. (2010). Dengue virus inhibits the production of type I interferon in primary human dendritic cells. J. Virol. 84: 4845-4850.

Rose P W, Beran B, Bi C, Bluhm W F, et al. (2011). The RCSB Protein Data Bank: redesigned web site and web services. Nucleic Acids Res. 39 (Suppl 1): D392-D401.

Santelli E, Leone M, Li C, Fukushima T, et al. (2005). Structural analysis of Siah1-Siah-interacting protein interactions and insights into the assembly of an E3 ligase multiprotein complex. J. Biol. Chem. 280: 34278-34287.

Schindler C, Levy DE and Decker T (2007). JAK-STAT signaling: from interferons to cytokines. J. Biol. Chem. 282: 20059-20063.

Schneider TD and Stephens RM (1990). Sequence logos: a new way to display consensus sequences. Nucleic Acids Res. 18: 6097-6100.

Sneath P and Sokal R (1973). Numerical taxonomy. WF Freeman and Co., San Francisco.

Stark GR, Kerr IM, Williams BR, Silverman RH, et al. (1998). How cells respond to interferons. Ann. Rev. Biochem. 67: 227-264.

Stebbins JL, Santelli E, Feng Y, De SK, et al. (2013). Structure-based design of covalent siah inhibitors. Chem. Biol. 20: 973-982.

Tamura K, Stecher G, Peterson D, Filipski A, et al. (2013). MEGA6: molecular evolutionary genetics analysis version 6.0. Mol. Biol. Evol. 30: 2725-2729.

Tasaki T, Mulder LC, Iwamatsu A, Lee MJ, et al. (2005). A family of mammalian E3 ubiquitin ligases that contain the UBR box motif and recognize N-degrons. Mol. Cell. Biol. 25: 7120-7136.

The UniProt Consortium (2008). The universal protein resource (UniProt). Nucleic Acids Res. 36 (Suppl 1): D190-D195.

Tsai Y-T, Chang S-Y, Lee C-N and Kao C-L (2009). Human TLR3 recognizes dengue virus and modulates viral replication in vitro. Cell. Microbiol. 11: 604-615.

Vinkemeier U, Cohen S, Moarefi I, Chait B, et al. (1996). DNA binding of in vitro activated Stat1 alpha, Stat1 beta and truncated Stat1: interaction between NH2-terminal domains stabilizes binding of two dimers to tandem DNA sites. The EMBO J. 15: 5616.

Wang J, Huo K, Ma L, Tang L, et al. (2011). Toward an understanding of the protein interaction network of the human liver. Mol. Syst. Biol. 7: 536.

Wiederstein M and Sippl MJ (2007). ProSA-web: interactive web service for the recognition of errors in three-dimensional 
structures of proteins. Nucleic Acids Res. 35 (Suppl 2): W407-W410.

Yap TL, Xu T, Chen Y-L, Malet H, et al. (2007). Crystal structure of the dengue virus RNA-dependent RNA polymerase catalytic domain at 1.85-angstrom resolution. J. Virol. 81: 4753-4765.

Zuckerkandl E and Pauling L (1965). Evolutionary divergence and convergence in proteins. Evolving Genes Proteins 97 : $97-166$. 